\title{
Molecular Dynamics Studies on the Effects of Water Speciation on Interfacial Structure and Dynamics in Silica-Filled PDMS Composites
}

\author{
R. H. Gee, R. S. Maxwell, L. N. Dinh, B. Balazs
}

This article was submitted to

U.S. Department of Energy

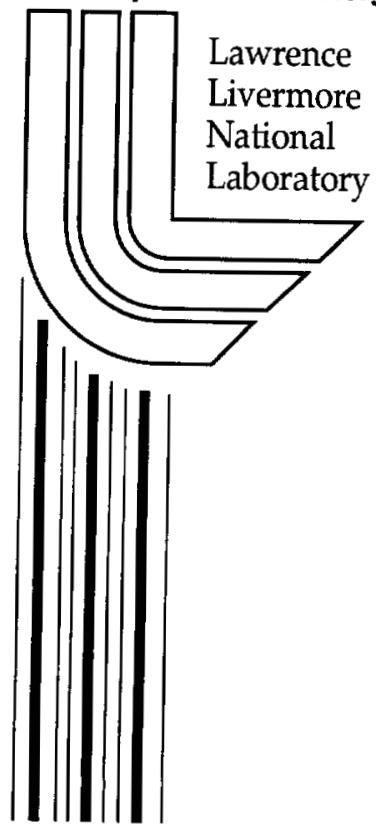

2001 Materials Research Society Fall Meeting, Boston, MA., November 26-30, 2001

November 21, 2001 


\section{DISCLAIMER}

This document was prepared as an account of work sponsored by an agency of the United States Government. Neither the United States Government nor the University of California nor any of their employees, makes any warranty, express or implied, or assumes any legal liability or responsibility for the accuracy, completeness, or usefulness of any information, apparatus, product, or process disclosed, or represents that its use would not infringe privately owned rights. Reference herein to any specific commercial product, process, or service by trade name, trademark, manufacturer, or otherwise, does not necessarily constitute or imply its endorsement, recommendation, or favoring by the United States Government or the University of California. The views and opinions of authors expressed herein do not necessarily state or reflect those of the United States Government or the University of California, and shall not be used for advertising or product endorsement purposes.

This is a preprint of a paper intended for publication in a journal or proceedings. Since changes may be made before publication, this preprint is made available with the understanding that it will not be cited or reproduced without the permission of the author.

This work was performed under the auspices of the United States Department of Energy by the University of California, Lawrence Livermore National Laboratory under contract No. W-7405-Eng-48.

This report has been reproduced directly from the best available copy.

Available electronically at http://www.doc.gov/bridge

Available for a processing fee to U.S. Department of Energy

And its contractors in paper from

U.S. Department of Energy

Office of Scientific and Technical Information

P.O. Box 62

Oak Ridge, TN 37831-0062

Telephone: (865) 576-8401

Facsimile: (865) 576-5728

E-mail: reports@adonis.osti.gov

Available for the sale to the public from

U.S. Department of Commerce

National Technical Information Service

5285 Port Royal Road

Springfield, VA 22161

Telephone: (800) 553-6847

Facsimile: (703) 605-6900

E-mail: orders@ntis.fedworld.gov

Online ordering: http://www.ntis.gov/ordering.htm

\section{OR}

Lawrence Livermore National Laboratory

Technical Information Department's Digital Library

http://www.llnl.gov/tid/Library.html 


\title{
Molecular Dynamics Studies on the Effects of Water Speciation on Interfacial Structure and Dynamics in Silica-Filled PDMS Composites.
}

\author{
Richard H. Gee, Robert S. Maxwell, Long N. Dinh, Bryan Balazs \\ Chemistry and Materials Science Directorate \\ Lawrence Livermore National Laboratory \\ Livermore, CA 94551, U.S.A.
}

\begin{abstract}
Significant changes in materials properties of siloxane based polymers can be obtained by the addition of inorganic fillers. In silica-filled polydimethylsiloxane (PDMS) based composites the mechanism of this reinforcing behavior is presumably hydrogen bonding between surface hydroxyls and backbone siloxane species. We have chosen to investigate in detail the effect of chemisorbed and physisorbed water on the interfacial structure and dynamics in silica-filled PDMS based composites. Toward this end, we have combined molecular dynamics simulations and experimental studies employing DMA and NMR analysis. Our results suggest that the polymer-silica contact distance and the mobility of interfacial polymer chains significantly decreased as the hydration level at the interface was reduced. The reduced mobility of the PDMS chains in the interfacial domain reduced the overall, bulk, motional properties of the polymer, thus causing an effective "stiffening" of the polymer matrix. The role of the long-ranged Coulombic interactions on the structural features and chain dynamics of the polymer were also examined. Both are found to be strongly influenced by the electrostatic interactions as identified by the bond orientation time correlation function and local density distribution functions. These results have important implications for the design of nanocomposite silica-siloxane materials.
\end{abstract}

\section{INTRODUCTION}

Silica-filled polydimethylsiloxane (PDMS) composite systems are of broad appeal due to their chemical and environmental resilience and the availability of a wide range of tailorable chemical and mechanical properties[1-3]. This versatility is due, at least in part, to the presence of inorganic filler materials which are well know to significantly alter polymer material mechanical properties. Understanding of the interfacial chemistry is important for the ultimate engineering of mechanical, electrical, or chemical properties. The reinforcing mechanisms that control the material property changes in polymer-silica composite materials, for example, are poorly understood and rational control of the mechanical properties is difficult and often based solely on empirical correlations. With the large financial investment by the polymer industry in filled polymers and the increased interest in organic-inorganic nanocomposite materials, in-depth studies of interfacial interactions between filler surfaces and associated polymer chains are warranted.

The primary reinforcing mechanism by which silica fillers are thought to alter the properties of siloxane based polymeric materials is via hydrogen bonding interactions. In an effort to gain further insight into the reinforcing mechanisms of inorganic fillers on PDMS based composites, we have initiated a computational study of the effects of changes in interfacial 
hydroxyl content on the chain dynamics of adsorbed PDMS chains. The data obtained in this study have implications on rational engineering of PDMS-silica materials, chemical aging of composite materials, and future design of nano-scale inorganic-organic composite materials. In this paper, we use molecular dynamics (MD) to study the effects of fillers on a PDMS polymer at the molecular level, which provides an ideal opportunity for direct insight into the interfacial structure and dynamics.

\section{COMPUTATIONAL METHOD}

\section{Polymer-Filler Representation}

The MD simulations of PDMS silica composites were carried out using 3-dimensional (3D) cubic periodic boundary conditions. The model PDMS consists of a single chain of a 120 dimethylsiloxane monomer units. The use of single chain polymers to represent amorphous melts and glasses is common, and has proven to be quite accurate in replicating the behavior of experimental polymeric systems[4]. The PDMS chain was then interfaced onto a fumed silica surface with the approximate dimensions of $22 \AA \times 22 \AA$ and thickness of $15 \AA$. The actual cell length normal to the silica surface was chosen such that the PDMS interfaced to the silica surface did not interact with its periodic image. The PDMS melt therefore experiences both a solid and vacuum interface. The number of surface hydroxyl groups found on the silica surface was also varied so as to allow for the direct elucidation of the effects of hydrogen bonding interactions between the polymer and filler surface. The hydroxyl content ranged between that of a dehydrated silica surface (zero hydroxyl groups) to one with twice the average number of groups per unit area, as determined experimentally[5] (i.e., $\approx 8 \mathrm{OH} / 100 \AA^{2}$ ). Simulations were also performed with an explicit layer of free physisorbed water placed at the PDMS / silica interface. All atoms in the system were treated explicitly.

The initial polymer starting configurations were generated using a Monte Carlo method[6]. The monomers were allowed to 'polymerize' in a head-to-tail manner with no monomer reversals. The resulting amorphous structure along with the silica surface was then relaxed by energy minimization.

\section{Potential Functions}

The MD simulations of the polymer silica composite employ the CFF91 force field parameter set [7, 8]. The CFF91 potential consists of valence terms including diagonal and off diagonal cross coupling terms, and non-bonded van der Waals and Coulombic interaction terms. The functional form of the potential employs a quartic polynomial for bond stretching and angle bending and a three-term Fourier expansion for torsions. The out-of-plane coordinate was defined according to Wilson et al. [9]. The atomic charges for the non-bonded Coulombic interaction are derived from ab initio calculations. The van der Waals interactions were implemented using the well known Lennard-Jones (LJ) potential, making use of an inverse 9thpower term for the repulsive part rather than the more customary 12th-power term.

All valence degrees of freedom were explicitly treated and unconstrained. Non-bonded interactions were truncated for atom pairs with an inter-atomic distance greater than $9.5 \AA$. The truncation was implemented using a fifth-order spline between $8.5 \AA$ and $9.5 \AA$ to avoid 
discontinuities in the energies at the cutoff. A buffer width of $0.5 \AA$ was used in the generation of the neighbor list. The long-range electrostatic interactions in the system were calculated employing the standard Ewald summation technique[10].

\section{Molecular Dynamics Method}

The polymer silica composite simulations were performed using constant particle number, volume, and temperature $(N V T)$ dynamics. The Verlet velocity[10] time integration method was used with a time step of $0.5 \mathrm{fs}$. The Andersen[11] method was used for constant $N V T$ dynamics. All structures were initially simulated at $550 \mathrm{~K}$ for a minimum duration of $1 \mathrm{~ns}$ so as to equilibrate the system, followed by a data collection period of a minimum of the same.

All computations were carried out with the DISCOVER commercial software program from Molecular Simulations, Inc.[6], The computations were performed on Silicon Graphics Octane R12000 workstations.

\section{EXPERIMENTAL}

Polymer chain dynamics were measured using a 1H NMR spin-echo approach that has been described previously $[12,13]$. A small sample of filled polymer was sealed in a $5 \mathrm{~mm}$ NMR tube with a small amount of desiccant and allowed to age for up to 1 year. Modal analysis experiments were performed by placing a layer of filled polymer composite in a sealed canister with a layer of desiccant and a stainless steel mass, and measuring the resonant frequency of this setup at specific intervals.

\section{RESULTS AND DISCUSSION}

We first examine the local density distribution of the PDMS melt as a function of distance from the silica surface (Figure 1) at $550 \mathrm{~K}$. The local density is a convenient quantitative measure of the self-arrangement of the PDMS melt. The effects of silica hydration are apparent in the overall polymer structure. Tall and narrow first and second fluid layers are observed in the immediate vicinity of the substrate that reflects localized ordering of the polymer at the hydrated silica surface (Figure 1a), however, the local ordering gradually diminishes the hydration level of the silica surface such that the distinct first and second fluid layers are no longer apparent when interfaced to the dehydrated silica surface (Figure 1b). The contact distance (distance between polymer and surface) is also found to decrease as the hydration level is decreased.

The role of the long-ranged Coulombic interactions in determining the structure and overall interactions between polymer and filler is of interest. Moreover, studies such as this provide valuable insight into the 'tuneability' of the polymer material properties. To this end, simulations on the above systems have been performed with all the atomic charges set to zero. The resultant local density distributions were altered as compared to their fully charged counterparts in that the overall structure was seen to diminish. The bifurcation seen in the first fluid layer in the local structure of the oxygen moiety of the polymer backbone (Figure 1a) is seen merged into a single peak in the non-Coulombic analogue. Additionally, the contact 

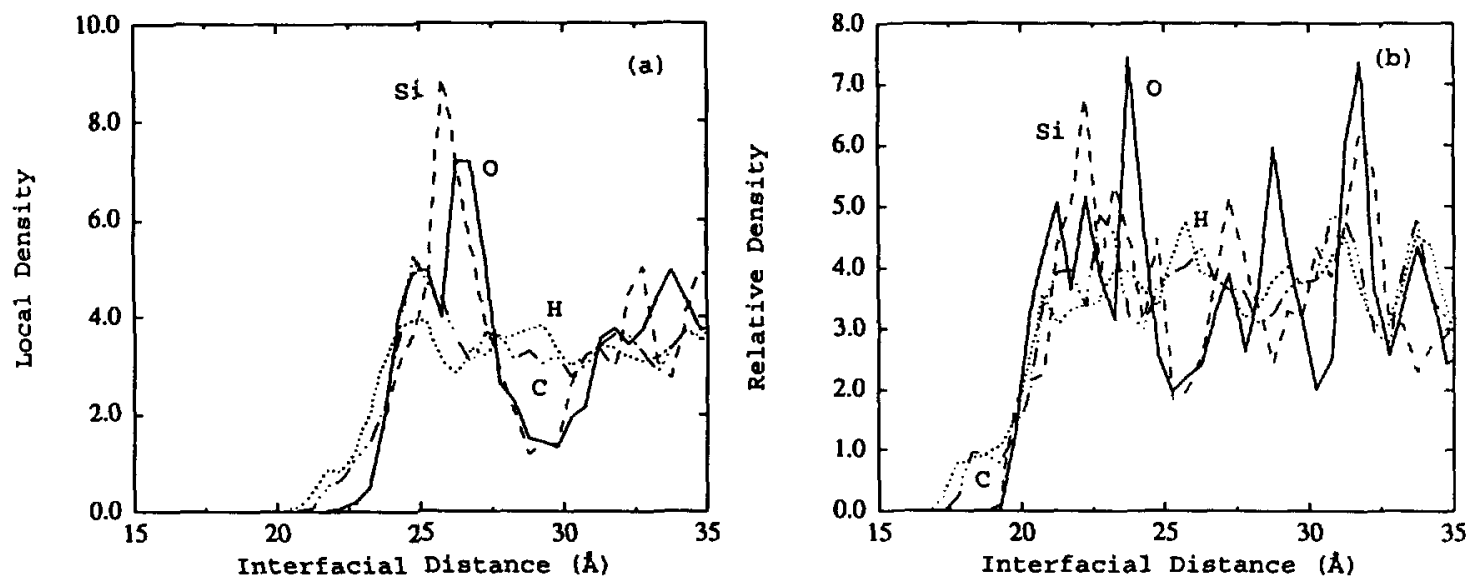

Figure 1. Local density profiles of PDMS with surface hydration levels of $\sim 8 \mathrm{OH} / 100 \AA^{2}$ (a) and dehydrated (b) at $550 \mathrm{~K}$. Lines are labeled in the figure.

distance of the non-Coulombic systems were reduced as compared to the charge analogues. The contact distance as a function of decreased hydration was also seen. The structural difference seen between Coulombic and non-Coulombic systems are consistent with the notion that the polymer-filler interactions are largely dominated by the long-ranged hydrogen bonding interactions and not solely based on the geometric constraints of packing the polymer close to the surface.

In hopes of elucidating the influence of the silica filler on the PDMS dynamics, we next turn our attention to the local dynamics of the polymer both as a function of hydration level of the silica surface as well as the distance from its surface. Among the various types of local motion found in polymer liquids, the change in the orientation of individual bonds with time is one that can be readily evaluated from the MD simulations. The bond reorientation motions are also amenable to experimental measurements such as NMR or modal analysis. Here we investigate the PDMS motional dynamics using the following time autocorrelation function (ACF),

$$
P_{1}(t)=\{\mu(0) \bullet \mu(t)\rangle
$$

where $\mu(t)$ is a unit vector and \langle\rangle denotes an ensemble average over all such vectors. In particular, we have investigated the bond vector ACF between the methyl and backbone carbon atoms $\left(C-\mathrm{CH}_{3}\right)$. Figure 2 shows $P_{1}(t)$ for PDMS at $550 \mathrm{~K}$ as a function of both the distance from the silica surface (Figure $2 \mathrm{a}$ ) at a surface hydration level of $\sim 8 \mathrm{OH} / 100 \AA^{2}$ and hydration level of the surface for those bonds near the surface (Figure $2 b$ ). It is readily apparent from Figure 2a that the relaxation near the surface is slowed considerably as compared to those bonds found further from the surface (bulk). The PDMS dynamics are also found to be affected by the hydration levels of the silica surface. The general trend is that the relaxation times tend to increase as the hydration level decreases, thus effectively "stiffening" the polymer matrix. This may be in part 

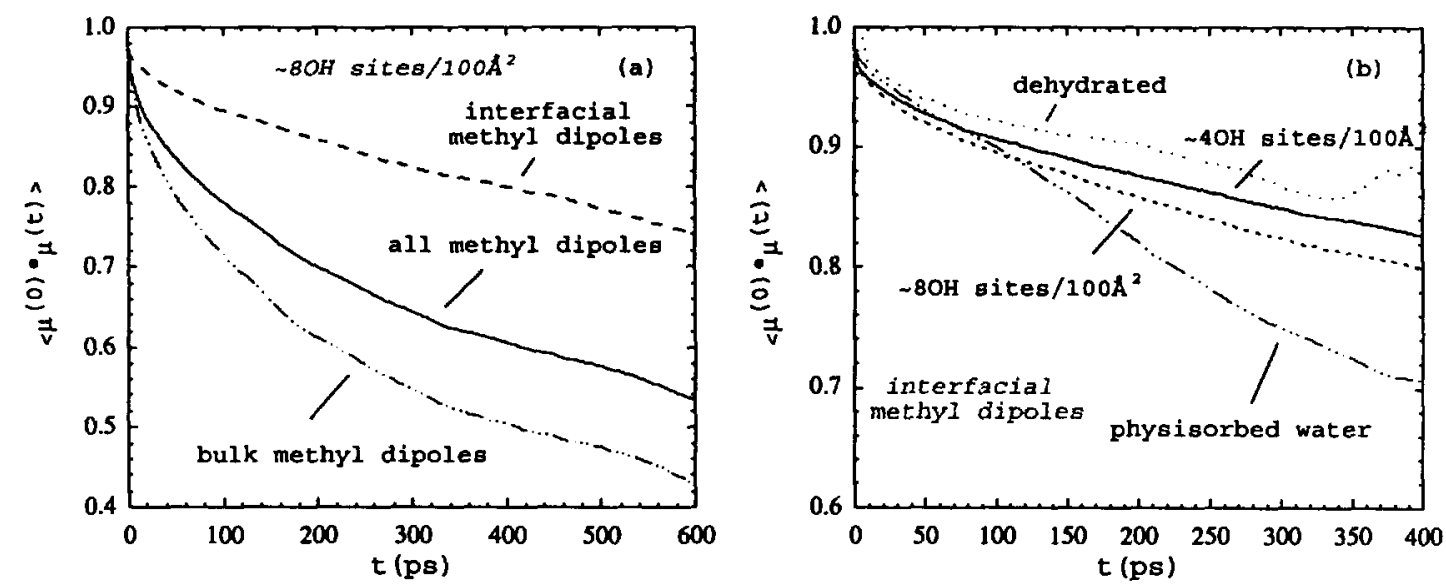

Figure 2. The decay of $P_{3}(t)$ at $550 \mathrm{~K}$. Panel a shows decay for those PDMS $\mathrm{C}-\mathrm{CH}_{3}$ bonds closest to the silica surface as a function of surface hydration, where panel $b$ shows decay as a function of contact distance at a hydration level of $\sim 8 \mathrm{OH} / 100 \AA^{2}$.. Lines are labeled in the figure.

due to screening of the long-ranged electrostatic charge by the hydroxyl groups or free physisorbed water in the interfacial region. To test this assumption, relaxation dynamics of noncharged analogues were studied. The results show that the PDMS relaxation dynamics are slowed by roughly an order of magnitude at $550 \mathrm{~K}$ in the presence of the long-ranged electrostatic interactions.

Figure 3 shows our simulation results on the motional dynamic of PDMS as a function of hydration level of the silica filler and compared to both the NMR and modal analysis results described in the experimental section of this paper. The relative "stiffness" of the PDMS matrix is found to increase as the hydration level decreases as seen both experimentally and in our simulation results presented here.

\section{CONCLUSIONS}

Our simulations reveal that the polymer-silica contact distance is significantly decreased as the level of "water" in the interfacial region is decreased. We also find that the "water" in the interfacial region seems to effectively "screen" the long-ranged interactions. We therefore suggest here the closer polymer-silica contact and the loss of electrostatic "screening" upon dehydration of the silica filler particles as a possible mechanism for polymer "stiffening" seen experimentally in such systems. 


\section{ACKNOWLEDGEMENTS}

This work was performed under the auspices of the U. S. Department of Energy by University of California Lawrence Livermore National Laboratory under contract No. W-7405Eng-48.
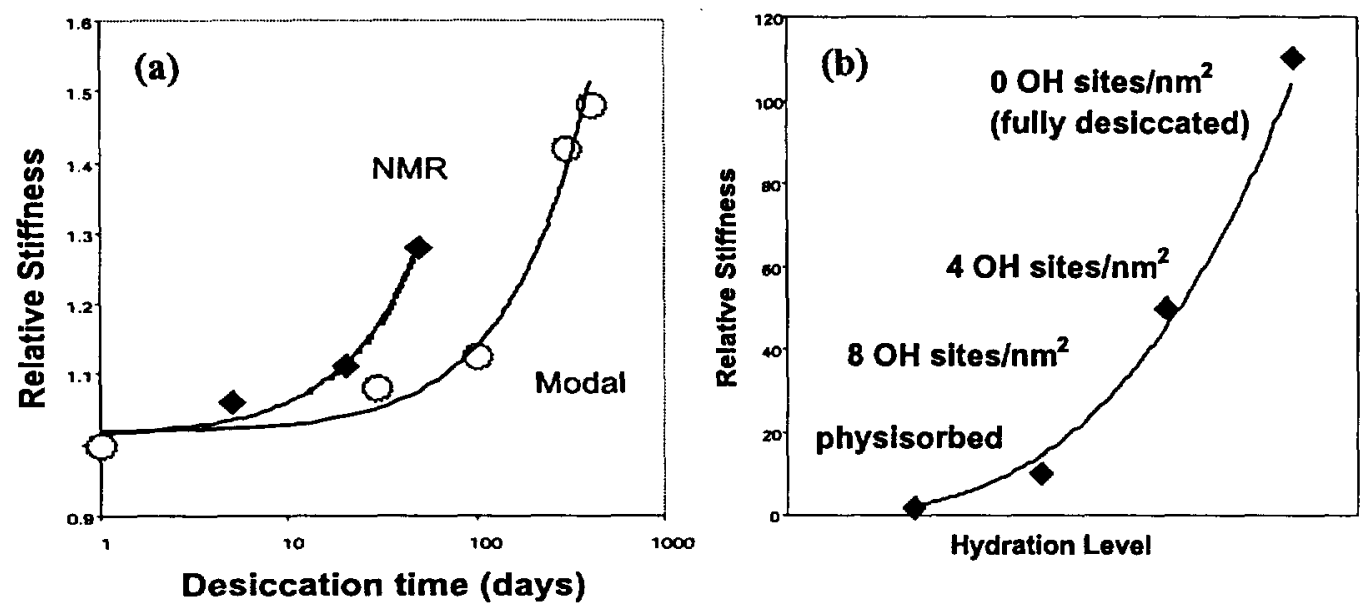

Figure 3. NMR and modal analysis (panel a) of changes in relative stiffness as a function of time undergoing chemical desiccation; changes in relative stiffness as measured by computational methods described in text due to changes in surface water and hydroxyl concentration (panel b).

\section{REFERENCES}

1. G. Kraus, Rubber Chem. Tech. 38, 1070 (1965).

2. J. M. Zeigher, F. W. G. Fearon, Silicon Based Polymer Science: A Comprehensive Resource, Advances in Chemistry (ACS Press, 1990), vol. 224.

3. P. Vondracek, A. Pouchelon, Rubber Chem. Technol. 63, 202 (1990).

4. R. H. Gee, L. E. Fried, R. C. Cook, Macromolecules 34, 3050 (2001).

5. D. Bordeaux, J. P. Cohen-Addad, Polymer 31, 743-748 (1990).

6. Discover molecular modeling software; Molecular Simulations, Inc.; San Diego, CA.

7. J. A. Maple et al., J. Comp. Chem. 15, 162 (1994).

8. J. R. Maple, U. Dinur, A. T. Hagler, Proc. Natl. Acad. Sci. 85, 5350 (1988).

9. E. B. Wilson, J. C. Decius, P. C. Cross, Molecular Vibrations (Dover, New York, 1980).

10. M. P. Allen, D. J. Tildesley, Computer Simulation of Liquids (Clarendon Press: Oxford, U.K., 1989).

11. H. C. Andersen, J. Chem. Phys. 72, 2384 (1980).

12. R. S. Maxwell, G. B. Balazs, J. Chem. Phys. (submitted).

13. A. Chien, R. S. Maxwell, D. Chambers, G. B. Balazs, Rad. Phys. Chem. 59, 493 (2000). 